\title{
Superconvergence of a New Nonconforming Mixed Finite Element Scheme for Elliptic Problem
}

\author{
Lifang Pei ${ }^{1,2}$ and Dongyang Shi ${ }^{2}$ \\ ${ }^{1}$ Department of Mathematics and Physics, Luoyang Institute of Science and Technology, Luoyang 471003, China \\ ${ }^{2}$ School of Mathematics and Statistics, Zhengzhou University, Zhengzhou 450001, China \\ Correspondence should be addressed to Dongyang Shi; shi_dy@zzu.edu.cn
}

Received 3 January 2013; Revised 30 June 2013; Accepted 5 July 2013

Academic Editor: Trung Nguyen Thoi

Copyright (c) 2013 L. Pei and D. Shi. This is an open access article distributed under the Creative Commons Attribution License, which permits unrestricted use, distribution, and reproduction in any medium, provided the original work is properly cited.

\begin{abstract}
A new nonconforming mixed finite element scheme for the second-order elliptic problem is proposed based on a new mixed variational form. It has the lowest degrees of freedom on rectangular meshes. The superclose property is proven by employing integral identity technique. Then global superconvergence result is derived through interpolation postprocessing operators. At last, some numerical experiments are carried out to verify the theoretical analysis.
\end{abstract}

\section{Introduction}

Mixed finite element method (MFEM) is an important branch of FEMs and has been used widely in numerical computation of practical problems. A lot of studies on this aspect have been devoted to the second-order elliptic problems [1-5], in which two approximation spaces of MFEM should satisfy the famous B-B condition [6]. However, since the variable $u$ and flux $\psi=\nabla u$ belong to $L^{2}(\Omega)$ and $H(\operatorname{div}, \Omega)$, respectively, it is not easy to construct a stable MFE space pair. In order to circumvent or ameliorate this deficiency, many approaches have been proposed, such as the least squares FEM [7], stabilization FEM [8], and $H^{1}$ Galerkin FEM [9]. Recently, [10] presented a new MFEM, in which B-B condition is automatically satisfied when $M_{h}$ and $H_{h}$ meet a relation of inclusion; that is, $\nabla M_{h} \subset H_{h}$, where $M_{h}$ and $H_{h}$ are finite element approximation spaces of $u$ and flux $\psi$, respectively. This advantage makes the construction of stable MFE space pair extremely simple and convenient. A family of triangular and rectangular conforming MFE space pairs with lower degrees of freedom is constructed in [10], in which the total degrees of freedom of first-order and secondorder MFE schemes are about $5 N_{p}$ and $16 N_{p}$, respectively; herein $N_{p}$ denotes the number of nodal points in subdivision. Later, [11] derived the similar results with [10] for conforming MFEM and gave a numerical example.
In this paper, motivated by the idea of $[10,11]$, we first construct a new nonconforming MFEM (NMFEM). The original variable $u$ is approximated by the constrained $Q_{1}^{\text {rot }}$ element space [12] and the flux $\psi$ by piecewise constant vectors space, respectively. Note that the total degrees of freedom of the NMFEM are only about $3 N_{p}$, the lowest on rectangular meshes. We prove that it satisfies B-B condition. Then by the use of integral identity technique, we derive the superclose property for $u$ in energy norm and flux $\psi$ in $L^{2}$ norm. Furthermore, the global superconvergence result with order $\mathrm{O}\left(h^{2}\right)$ is obtained through interpolation postprocessing operators. Finally, some numerical results are provided to verify the theoretical analysis. It is observed that, compared with FEM using 4-node quadrilateral (FEM-Q4) and MFEM ( $u$ and flux $\psi$ are approximated by piecewise constants and the Raviart-Thomas element, resp.), NMFEM behaves well and has higher rates than MFEM, and it has almost the same rate as FEM-Q4 for $u$ in $L^{2}$ norm, but a higher rate than FEMQ4 for $u$ in energy norm. Moreover, NMFEM is effective and accurate for the diffusion problem studied in [13].

The remainder of this paper is organized as follows. In Section 2, we introduce NMFEM and derive the superclose property and superconvergence results. In Section 3, we carry out some numerical experiments to show the performance of NMFEM. 
We will use standard notations for the Sobolev spaces $H^{m}(\Omega)$ with norm $\|\cdot\|_{m}$ and seminorm $|\cdot|_{m}, H^{m}(K)$ with norm $\|\cdot\|_{m, K}$ and seminorm $|\cdot|_{m, K}$, where $m>0$ is an integer. Besides, let $\|\cdot\|_{0}$ and $\|\cdot\|_{0, K}$ be the $L^{2}(\Omega)$ norm and $L^{2}(K)$ norm, respectively. Throughout the paper, $C$ denotes a positive constant independent of the mesh parameter $h$ and may be different at each appearance.

\section{Superconvergence Analysis for NMFEM}

Consider the following elliptic problem:

$$
\begin{gathered}
-\Delta u=f, \quad \text { in } \Omega, \\
u=0, \quad \text { on } \partial \Omega,
\end{gathered}
$$

where $\Omega \subset R^{2}$ is a bounded convex polygon domain, $f \in$ $L^{2}(\Omega)$.

Let $\psi=\nabla u$, and then problem (1) is equivalent to the following equations:

$$
\begin{gathered}
\psi-\nabla u=0, \quad \text { in } \Omega, \\
-\operatorname{div} \psi=f, \quad \text { in } \Omega, \\
u=0, \quad \text { on } \partial \Omega .
\end{gathered}
$$

We adopt a new mixed variational form in [10] of problem (2). Find $(\psi, u) \in H \times M$ such that

$$
\begin{gathered}
a(\psi, \varphi)+b(\varphi, u)=0, \quad \forall \varphi \in H, \\
b(\psi, v)=G(v), \quad \forall v \in M,
\end{gathered}
$$

where $H=\left(L^{2}(\Omega)\right)^{2}, M=H_{0}^{1}(\Omega), a(\psi, \varphi)=\int_{\Omega} \psi \cdot \varphi d x d y$, $b(\varphi, v)=-\int_{\Omega} \varphi \cdot \nabla v d x d y, G(v)=-\int_{\Omega} f v d x d y$.

Obviously, $a(\cdot, \cdot)$ and $b(\cdot, \cdot)$ are continuous bilinear functionals, $G(\cdot)$ is a continuous linear functional, and for all $\varphi \in$ $H, a(\varphi, \varphi)=\int_{\Omega} \varphi \cdot \varphi d x d y=\|\varphi\|_{0}^{2}$. Moreover, for $v \in M$, we have $\nabla v \in H$. So there exists a constant $\beta>0$ such that

$$
\sup _{\varphi \in H} \frac{b(\varphi, v)}{\|\varphi\|_{0}} \geq \frac{b(-\nabla v, v)}{\|-\nabla v\|_{0}}=|v|_{1} \geq \beta\|v\|_{1},
$$

that is, the B-B condition is satisfied, and therefore (3) has a unique solution $(\psi, u)$.

Let $Q((\psi, u),(\varphi, v))=a(\psi, \varphi)+b(\varphi, u)-b(\psi, v)$, and then (3) can be written as

$$
Q((\psi, u),(\varphi, v))=-G(v), \quad \forall \varphi \in H, v \in M \text {. }
$$

Let $T_{h}$ be a rectangular partition of the domain $\Omega$. For a given element $K \in T_{h}$, its four vertices are denoted by $a_{1}=$ $\left(x_{K}-h_{x}, y_{K}-h_{y}\right), a_{2}=\left(x_{K}+h_{x}, y_{K}-h_{y}\right), a_{3}=\left(x_{K}+h_{x}, y_{K}+\right.$ $\left.h_{y}\right), a_{4}=\left(x_{K}-h_{x}, y_{K}+h_{y}\right)$, and four edges by $l_{i}=\overline{a_{i} a_{i+1}}(i=$ $1,2,3,4 \bmod 4), h_{K}=\max _{K \in T_{h}}\left\{h_{x}, h_{y}\right\}, h=\max _{K \in T_{h}} h_{K}$. Let $\widehat{K}=[-1,1] \times[-1,1]$ be the reference element with nodes $\widehat{a}_{1}=(-1,-1), \widehat{a}_{2}=(1,-1), \widehat{a}_{3}=(1,1), \widehat{a}_{4}=(-1,1)$ and edges $\widehat{l}_{i}=\overline{\hat{a}_{i} \widehat{a}_{i+1}}(i=1,2,3,4 \bmod 4)$.
Define the affine mapping $F: \widehat{K} \rightarrow K$ by

$$
\begin{aligned}
& x=x_{K}+h_{x} \xi, \\
& y=x_{K}+h_{y} \eta .
\end{aligned}
$$

Let $P_{1}(\widehat{K})$ be the space of polynomials with degrees $\leq 1$ defined on $\widehat{K}$, and then the constrained $Q_{1}^{\text {rot }}$ element space $\mathrm{CR}_{0}^{h}$ is defined by [12]:

$$
\begin{aligned}
\mathrm{CR}_{0}^{h}=\left\{v ;\left.v\right|_{K}=\widehat{v} \circ F^{-1}, \widehat{v} \in P_{1}(\widehat{K}),\right. \\
\left.\quad \int_{l}[v] d s=0, l \subset \partial K, \forall K \in T_{h}\right\},
\end{aligned}
$$

where $[v]$ denotes the jump value of $v$ across the boundary $l$, and $[v]=v$ if $l \subset \partial \Omega$.

Let $N_{i}^{V}$ denote the number of interior nodes. It has been proven in [12] that $\operatorname{dim}\left(\mathrm{CR}_{0}^{h}\right)=N_{i}^{V}$ and $P_{1}(\widehat{K})=\operatorname{span}\left\{\widehat{\phi}_{i}, \quad i=\right.$ $1 \sim 4\}$, where $\widehat{\phi}_{i}$ are defined associated with nodes $\widehat{a}_{i}(i=$ $1,2,3,4)$ of $\widehat{K}$ as

$$
\begin{array}{ll}
\widehat{\phi}_{1}=\frac{1}{4}(1-\xi-\eta), & \widehat{\phi}_{2}=\frac{1}{4}(1+\xi-\eta), \\
\widehat{\phi}_{3}=\frac{1}{4}(1+\xi+\eta), & \widehat{\phi}_{4}=\frac{1}{4}(1-\xi+\eta) .
\end{array}
$$

We choose the following $\mathrm{FE}$ spaces $M_{h}$ and $H_{h}$ to approximate $M$ and $H$, respectively:

$$
\begin{gathered}
M_{h}=\mathrm{CR}_{0}^{h}, \\
H_{h}=\left\{q=\left(q_{1}, q_{2}\right) ;\left.q\right|_{K} \in\left(Q_{0}(K)\right)^{2}, \forall K \in T_{h}\right\},
\end{gathered}
$$

where $Q_{0}(K)$ is the space of constants on $K$. Obviously, the total degree of freedoms of the nonconforming MFE space pair $H_{h} \times M_{h}$ is $3 N_{p}$, and $\|\cdot\|_{h}=\left(\sum_{K \in T_{h}}|\cdot|_{1, K}^{2}\right)^{1 / 2}$ is a norm over $M_{h}$.

Then the MFE approximation of problem (3) is to find $\left(\psi_{h}, u_{h}\right) \in H_{h} \times M_{h}$ such that

$$
\begin{gathered}
a\left(\psi_{h}, \varphi_{h}\right)+b_{h}\left(\varphi_{h}, u_{h}\right)=0, \quad \forall \varphi_{h} \in H_{h}, \\
b_{h}\left(\psi_{h}, v_{h}\right)=G\left(v_{h}\right), \quad \forall v_{h} \in M_{h},
\end{gathered}
$$

where $b_{h}\left(\varphi_{h}, v_{h}\right)=-\sum_{K \in T_{h}} \int_{K} \varphi_{h} \cdot \nabla v_{h} d x d y$.

For $v_{h} \in M_{h}$, from the affine mapping $F$ and the definition of $M_{h}$, we can get $\left.v_{h}\right|_{K} \in P_{1}(K)$, and then $\left.\nabla v_{h}\right|_{K} \in\left(Q_{0}(K)\right)^{2}$, that is, $\nabla v_{h} \in H_{h}$. Thus

$$
\begin{aligned}
\sup _{\varphi_{h} \in H_{h}} & \frac{b_{h}\left(\varphi_{h}, v_{h}\right)}{\left\|\varphi_{h}\right\|_{0}} \geq \frac{b_{h}\left(-\nabla v_{h}, v_{h}\right)}{\left\|-\nabla v_{h}\right\|_{0}} \\
= & \frac{\sum_{K \in T_{h}} \int_{K} \nabla v_{h} \cdot \nabla v_{h} d x d y}{\left\|-\nabla v_{h}\right\|_{0}}=\frac{\left\|v_{h}\right\|_{h}^{2}}{\left\|v_{h}\right\|_{h}}=\left\|v_{h}\right\|_{h},
\end{aligned}
$$

that is, the discrete B-B condition holds, and (10) has a unique solution $\left(\psi_{h}, u_{h}\right)$.

For all $\left(q_{h}, \lambda_{h}\right) \in H_{h} \times M_{h}$, let $Q_{h}\left(\left(q_{h}, \lambda_{h}\right),\left(\varphi_{h}, v_{h}\right)\right)=$ $a\left(q_{h}, \varphi_{h}\right)+b_{h}\left(\varphi_{h}, \lambda_{h}\right)-b_{h}\left(q_{h}, v_{h}\right)$, and then we can prove the following two important lemmas. 
Lemma 1. For all $\left(q_{h}, \lambda_{h}\right) \in H_{h} \times M_{h}$, the following inequality holds:

$$
\sup _{\left(\varphi_{h}, v_{h}\right) \in H_{h} \times M_{h}} \frac{Q_{h}\left(\left(q_{h}, \lambda_{h}\right),\left(\varphi_{h}, v_{h}\right)\right)}{\left\|\varphi_{h}\right\|_{0}+\left\|v_{h}\right\|_{h}} \geq C\left(\left\|q_{h}\right\|_{0}+\left\|\lambda_{h}\right\|_{h}\right) .
$$

Proof. In order to prove (12), we will construct a pair $\left(\varphi_{h}, v_{h}\right) \in H_{h} \times M_{h}$ satisfying

$$
Q_{h}\left(\left(q_{h}, \lambda_{h}\right),\left(\varphi_{h}, v_{h}\right)\right) \geq C\left(\left\|q_{h}\right\|_{0}+\left\|\lambda_{h}\right\|_{h}\right)\left(\left\|\varphi_{h}\right\|_{0}+\left\|v_{h}\right\|_{h}\right) .
$$

Obviously,

$$
Q_{h}\left(\left(q_{h}, \lambda_{h}\right),\left(q_{h}, \lambda_{h}\right)\right)=\left\|q_{h}\right\|_{0}^{2} .
$$

On the other hand, for a given arbitrary but fixed $\lambda_{h} \in M_{h}$, by the discrete B-B condition, there exists a $w_{h} \in H_{h}$ such that

$$
\begin{gathered}
\sum_{K \in T_{h}} \int_{K} w_{h} \cdot \nabla \lambda_{h} d x d y \geq\left\|w_{h}\right\|_{0}\left\|\lambda_{h}\right\|_{h}, \\
\left\|w_{h}\right\|_{0}=\left\|\lambda_{h}\right\|_{h} .
\end{gathered}
$$

So, for $0<r<1$, there holds

$$
\begin{aligned}
& Q_{h}\left(\left(q_{h}, \lambda_{h}\right),\left(-r w_{h}, 0\right)\right) \\
& \quad=-r a\left(q_{h}, w_{h}\right)+r \sum_{K \in T_{h}} \int_{K} w_{h} \cdot \nabla \lambda_{h} d x d y \\
& \quad \geq-r\left\|q_{h}\right\|_{0}\left\|\lambda_{h}\right\|_{h}+r\left\|\lambda_{h}\right\|_{h}^{2} \\
& \quad \geq-r\left\|q_{h}\right\|_{0}^{2}+\frac{3}{4} r\left\|\lambda_{h}\right\|_{h}^{2} .
\end{aligned}
$$

As a result, setting $\left(\varphi_{h}, v_{h}\right)=\left(q_{h}-r w_{h}, \lambda_{h}\right)$, we have

$$
\begin{aligned}
Q_{h}\left(\left(q_{h}, \lambda_{h}\right),\left(q_{h}-r w_{h}, \lambda_{h}\right)\right) \\
=Q_{h}\left(\left(q_{h}, \lambda_{h}\right),\left(q_{h}, \lambda_{h}\right)\right) \\
\quad+Q_{h}\left(\left(q_{h}, \lambda_{h}\right),\left(-r w_{h}, 0\right)\right) \\
\geq(1-r)\left\|q_{h}\right\|_{0}^{2}+\frac{3}{4} r\left\|\lambda_{h}\right\|_{h}^{2},
\end{aligned}
$$

which implies that

$$
\begin{gathered}
Q_{h}\left(\left(q_{h}, \lambda_{h}\right),\left(q_{h}-r w_{h}, \lambda_{h}\right)\right) \\
\geq C_{1}\left(\left\|q_{h}\right\|_{0}^{2}+\left\|\lambda_{h}\right\|_{h}^{2}\right) \\
\quad \geq \frac{C_{1}}{2}\left(\left\|q_{h}\right\|_{0}+\left\|\lambda_{h}\right\|_{h}\right)^{2},
\end{gathered}
$$

where $C_{1}=\min \{1-r,(3 / 4) r\}$.

Note that

$$
\begin{aligned}
\| q_{h}- & r w_{h}\left\|_{0}+\right\| \lambda_{h} \|_{h} \\
& \leq\left\|q_{h}\right\|_{0}+r\left\|w_{h}\right\|_{0}+\left\|\lambda_{h}\right\|_{h} \\
& =\left\|q_{h}\right\|_{0}+(1+r)\left\|\lambda_{h}\right\|_{h} \\
& \leq(1+r)\left(\left\|q_{h}\right\|_{0}+\left\|\lambda_{h}\right\|_{h}\right),
\end{aligned}
$$

and we have

$$
\begin{aligned}
& Q_{h}\left(\left(q_{h}, \lambda_{h}\right),\left(q_{h}-r w_{h}, \lambda_{h}\right)\right) \\
& \quad \geq \frac{C_{1}}{2(1+r)}\left(\left\|q_{h}\right\|_{0}+\left\|\lambda_{h}\right\|_{h}\right)\left(\left\|q_{h}-r w_{h}\right\|_{0}+\left\|\lambda_{h}\right\|_{h}\right),
\end{aligned}
$$

which follows the desired result (12).

Let $\Pi_{h}$ and $I_{h}$ denote the associated interpolation operators of $M_{h}$ and conforming bilinear element space, respectively. Let $\pi_{h}$ be the $Q_{1}^{\text {rot }}$ element interpolation operator [14]; that is, for all $v \in H^{1}(K), \pi_{h} v \in Q_{1}^{\text {rot }}$ satisfying $\int_{l} \pi_{h} v d s=\int_{l} v d s$, where $Q_{1}^{\text {rot }}=\left\{v_{h} ;\left.v_{h}\right|_{K} \in \operatorname{span}\left\{1, x, y, x^{2}-\right.\right.$ $\left.\left.y^{2}\right\}, \int_{l}\left[v_{h}\right] d s=0, l \subset \partial K\right\}$. It has been shown in [12] that, for all $v \in H^{2}(K), \Pi_{h} v=\pi_{h} I_{h} v \in M_{h}$ because $x y$ is a bubble function for $\pi_{h}$.

Lemma 2. Assume that $u \in H^{3}(\Omega), \psi \in\left(H^{2}(\Omega)\right)^{2}$, we have

$$
b_{h}\left(\psi-J_{h} \psi, v_{h}\right)=0, \quad \forall v_{h} \in M_{h} \text {, }
$$

$\sum_{K \in T_{h}} \int_{K} \nabla\left(u-\Pi_{h} u\right) \cdot \varphi_{h} d x d y \leq C h^{2}\|u\|_{3}\left\|\varphi_{h}\right\|_{0}, \quad \forall \varphi_{h} \in H_{h}$,

$$
\sum_{K \in T_{h}} \int_{\partial K} \psi \cdot n v_{h} d s \leq C h^{2}|\psi|_{2}\left\|v_{h}\right\|_{h}, \quad \forall v_{h} \in M_{h}
$$

where $J_{h}:\left(L^{2}(\Omega)\right)^{2} \rightarrow H_{h}$ is a interpolation operator satisfying $\int_{K}\left(\psi-J_{h} \psi\right) d x d y=0$.

Proof. Since (23) has been proven by one of the authors in [15], we only need to prove (21) and (22).

In fact, because $\partial v_{h} / \partial x$ and $\partial v_{h} / \partial y$ are constants on each $K \in T_{h}$, we have

$$
b_{h}\left(\psi-J_{h} \psi, v_{h}\right)=-\sum_{K \in T_{h}} \int_{K}\left(\psi-J_{h} \psi\right) \cdot \nabla v_{h} d x d y=0,
$$

which is (21).

On the other hand, note that

$$
\begin{aligned}
\int_{K} \nabla & \left(u-\Pi_{h} u\right) \cdot \varphi_{h} d x d y \\
= & \int_{K} \nabla\left(u-I_{h} u\right) \cdot \varphi_{h} d x d y \\
& +\int_{K} \nabla\left(I_{h} u-\Pi_{h} u\right) \cdot \varphi_{h} d x d y .
\end{aligned}
$$

Since $\varphi_{h}=\left(\varphi_{1 h}, \varphi_{2 h}\right)$ is a constant vector on $K$ and $\Pi_{h} v=$ $\pi_{h} I_{h} v$, it follows from integration by parts that

$$
\begin{aligned}
\int_{K} \nabla & \left(I_{h} u-\Pi_{h} u\right) \cdot \varphi_{h} d x d y \\
& =\left.\varphi_{h}\right|_{K} \int_{\partial K}\left(I_{h} u-\pi_{h} I_{h} u\right) d s \\
& =\left.\varphi_{h}\right|_{K} \int_{\partial K}\left(I_{h} u-I_{h} u\right) d s=0 .
\end{aligned}
$$


Furthermore, let $F(y)=(1 / 2)\left(\left(y-y_{K}\right)^{2}-h_{y}^{2}\right)$. Note that $\left(u-I_{h} u\right)\left(x_{K} \pm h_{x}, y_{K} \pm h_{y}\right)=0$ and $F\left(y_{K} \pm h_{y}\right)=0$. Employing integral identity technique [16], we have

$$
\begin{gathered}
\int_{K} \frac{\partial\left(u-I_{h} u\right)}{\partial x} \varphi_{1 h} d x d y \\
=\left.\varphi_{1 h}\right|_{K} \int_{K} F^{\prime \prime}(y) \frac{\partial\left(u-I_{h} u\right)}{\partial x} d x d y \\
=\left.\varphi_{1 h}\right|_{K}\left(F^{\prime}\left(y_{K}+h_{y}\right) \int_{l_{3}} \frac{\partial\left(u-I_{h} u\right)}{\partial x} d x\right. \\
-F^{\prime}\left(y_{K}-h_{y}\right) \int_{l_{1}} \frac{\partial\left(u-I_{h} u\right)}{\partial x} d x \\
\left.-\int_{K} F^{\prime}(y) \frac{\partial^{2}\left(u-I_{h} u\right)}{\partial x \partial y} d x d y\right) \\
=-\left.\varphi_{1 h}\right|_{K}\left(F\left(y_{K}+h_{y}\right) \int_{l_{3}} \frac{\partial^{2}\left(u-I_{h} u\right)}{\partial x \partial y} d x\right. \\
-F\left(y_{K}-h_{y}\right) \int_{l_{1}} \frac{\partial^{2}\left(u-I_{h} u\right)}{\partial x \partial y} d x \\
\left.-\int_{K} F(y) \frac{\partial^{3}\left(u-I_{h} u\right)}{\partial x \partial y^{2}} d x d y\right) \\
=\left.\varphi_{1 h}\right|_{K} \int_{K} F(y) \frac{\partial^{3}\left(u-I_{h} u\right)}{\partial x \partial y^{2}} d x d y \\
\leq C h^{2}\|u\|_{3, K}\left\|\varphi_{1 h}\right\|_{0, K} \cdot
\end{gathered}
$$

Similarly,

$$
\int_{K} \frac{\partial\left(u-I_{h} u\right)}{\partial y} \varphi_{2 h} d x d y \leq C h^{2}\|u\|_{3, K}\left\|\varphi_{2 h}\right\|_{0, K}
$$

Therefore, from (25)-(28), we have

$$
\begin{aligned}
& \sum_{K \in T_{h}} \int_{K} \nabla\left(u-\Pi_{h} u\right) \cdot \varphi_{h} d x d y \\
& =\sum_{K \in T_{h}} \int_{K} \nabla\left(u-I_{h} u\right) \cdot \varphi_{h} d x d y \\
& =\sum_{K \in T_{h}}\left(\int_{K} \frac{\partial\left(u-I_{h} u\right)}{\partial x} \varphi_{1 h} d x d y+\int_{K} \frac{\partial\left(u-I_{h} u\right)}{\partial y} \varphi_{2 h} d x d y\right) \\
& \leq C h^{2}\|u\|_{3}\left\|\varphi_{h}\right\|_{0} .
\end{aligned}
$$

The proof is completed.

Now we start to state the following superclose property.

Theorem 3. Assume that $(\psi, u)$ and $\left(\psi_{h}, u_{h}\right)$ are the solutions of (3) and (10), respectively; $u \in H^{3}(\Omega), \psi \in\left(H^{2}(\Omega)\right)^{2}$, there holds

$$
\left\|\psi_{h}-J_{h} \psi\right\|_{0}+\left\|u_{h}-\Pi_{h} u\right\|_{h} \leq C h^{2}\left(\|u\|_{3}+\|\psi\|_{2}\right) .
$$

Proof. For $\left(\varphi_{h}, v_{h}\right) \in H_{h} \times M_{h}$, from (2) and (10), we have

$$
\begin{aligned}
& a\left(\psi, \varphi_{h}\right)+b_{h}\left(\varphi_{h}, u\right)=a\left(\psi_{h}, \varphi_{h}\right)+b_{h}\left(\varphi_{h}, u_{h}\right)=0, \\
& b_{h}\left(\psi, v_{h}\right)-b_{h}\left(\psi_{h}, v_{h}\right) \\
&= b_{h}\left(\psi, v_{h}\right)-G\left(v_{h}\right) \\
&=-\sum_{K \in T_{h}} \int_{K} \psi \cdot \nabla v_{h} d x d y \\
&+\sum_{K \in T_{h}} \int_{K} f v_{h} d x d y \\
&=-\sum_{K \in T_{h}} \int_{\partial K} \psi \cdot n v_{h} d x d y \\
&+\sum_{K \in T_{h}} \int_{K}(\operatorname{div} \psi+f) v_{h} d x d y \\
&=-\sum_{K \in T_{h}} \int_{\partial K} \psi \cdot n v_{h} d x d y .
\end{aligned}
$$

Applying (31) yields

$$
\begin{aligned}
& Q_{h}\left(\left(\psi-\psi_{h}, u-u_{h}\right),\left(\varphi_{h}, v_{h}\right)\right) \\
& \quad=\sum_{K \in T_{h}} \int_{\partial K} \psi \cdot n v_{h} d x d y, \quad \forall\left(\varphi_{h}, v_{h}\right) \in H_{h} \times M_{h} .
\end{aligned}
$$

Using (12) in Lemma 1 and (32), we can obtain

$$
\begin{gathered}
\left\|\psi_{h}-J_{h} \psi\right\|_{0}+\left\|u_{h}-\Pi_{h} u\right\|_{h} \\
\leq C \sup _{\left(\varphi_{h}, v_{h}\right) \in H_{h} \times M_{h}} \frac{Q_{h}\left(\left(\psi_{h}-J_{h} \psi, u_{h}-\Pi_{h} u\right),\left(\varphi_{h}, v_{h}\right)\right)}{\left\|\varphi_{h}\right\|_{0}+\left\|v_{h}\right\|_{h}} \\
\leq \sup _{\left(\varphi_{h}, v_{h}\right) \in H_{h} \times M_{h}}\left(\left(Q_{h}\left(\left(\psi-J_{h} \psi, u-\Pi_{h} u\right),\left(\varphi_{h}, v_{h}\right)\right)\right.\right. \\
\left.-\sum_{K \in T_{h}} \int_{\partial K} \psi \cdot n v_{h} d x d y\right) \\
\left.\times\left(\left\|\varphi_{h}\right\|_{0}+\left\|v_{h}\right\|_{h}\right)^{-1}\right) \\
=\sup _{\left(\varphi_{h}, v_{h}\right) \in H_{h} \times M_{h}}\left(\left(a-J_{h} \psi, \varphi_{h}\right)+b_{h}\left(\varphi_{h}, u-\Pi_{h} u\right)\right. \\
-b_{h}\left(\psi-J_{h} \psi, v_{h}\right) \\
\left.\quad-\sum_{K \in T_{h}} \int_{\partial K} \psi \cdot n v_{h} d x d y\right) \\
\left.\times\left(\left\|\varphi_{h}\right\|_{0}+\left\|v_{h}\right\|_{h}\right)^{-1}\right) .
\end{gathered}
$$

Hence the desired result follows from the interpolation theorem and Lemma 2.

In order to derive global superconvergence for $u$ and flux $\psi$, we introduce the following postprocessing operators 


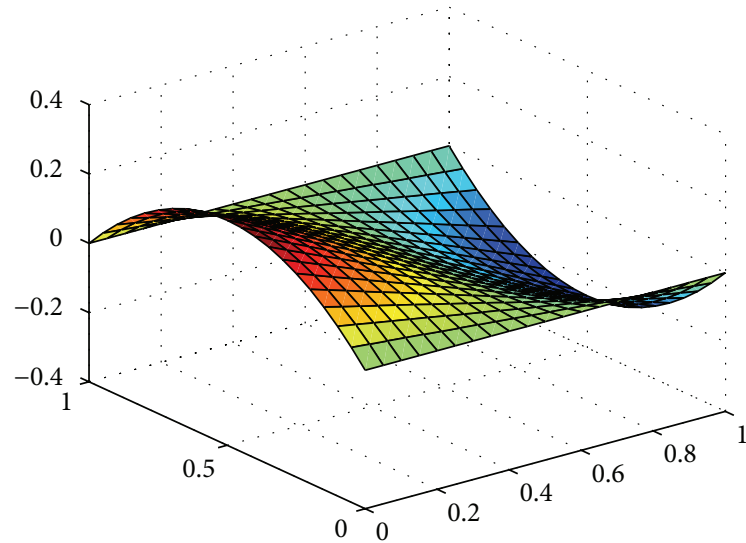

(a)

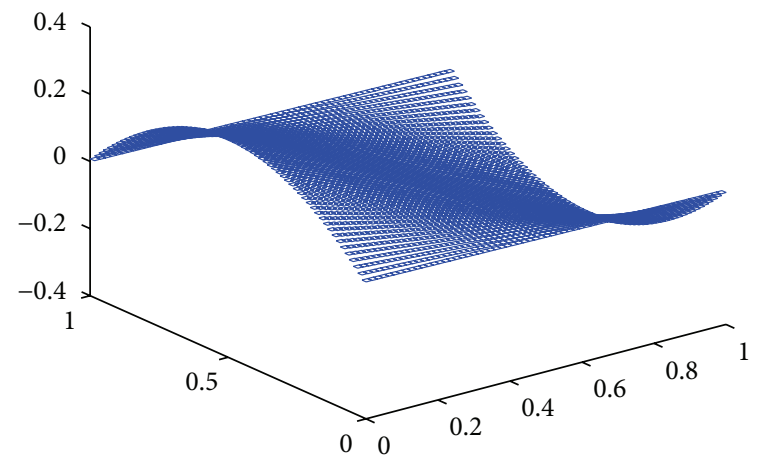

(b)

Figure 1: The exact flux $\psi_{1}$ (a) Numerical approximation $\psi_{1 h}$ (b).

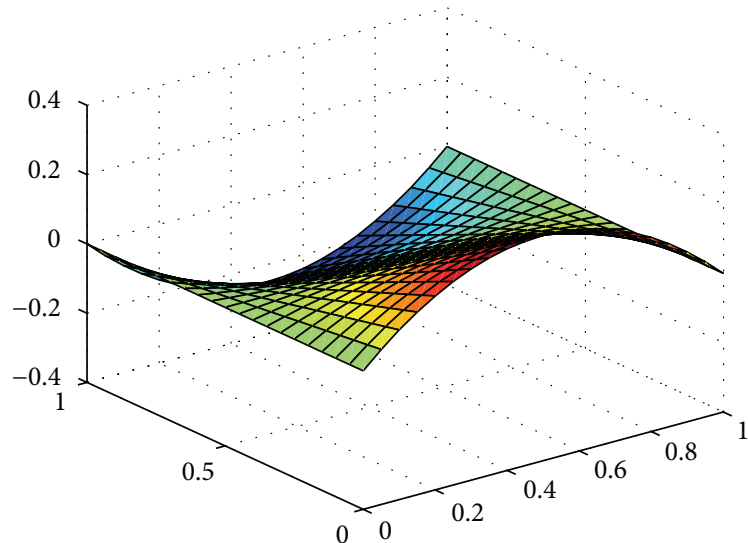

(a)

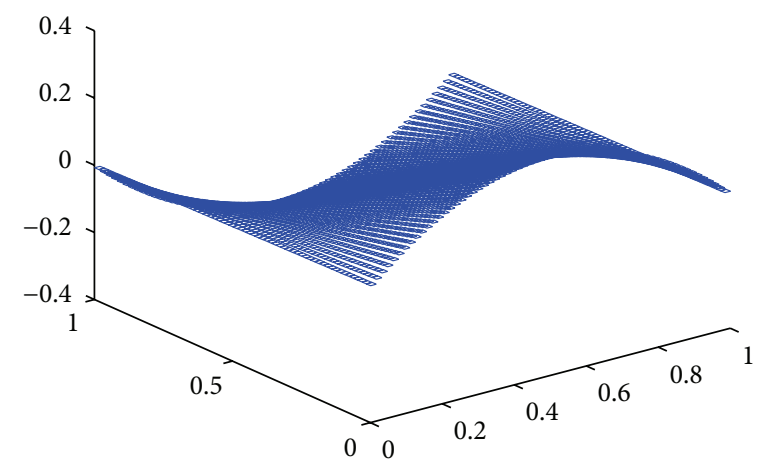

(b)

FIGURE 2: The exact flux $\psi_{2}$ (a) and numerical approximation $\psi_{2 h}$ (b).

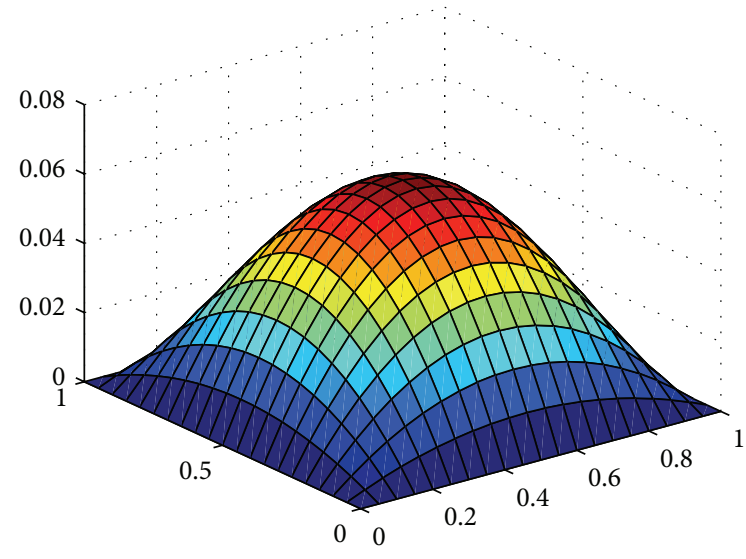

(a)

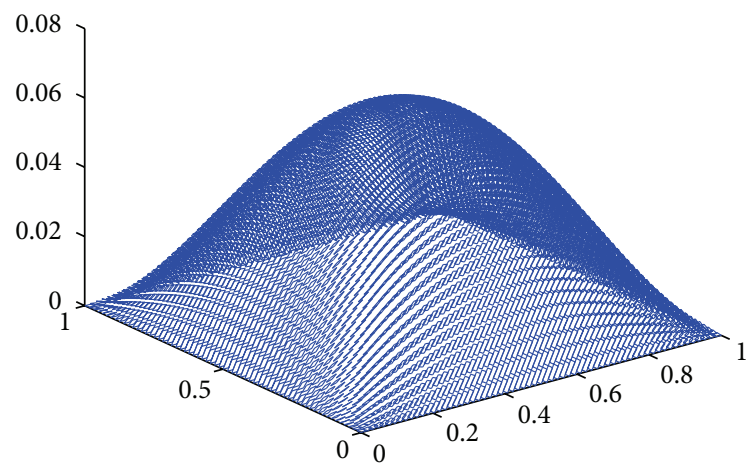

(b)

FIgURE 3: The exact displacement $u$ (a) and numerical approximation $u_{h}$ (b). 


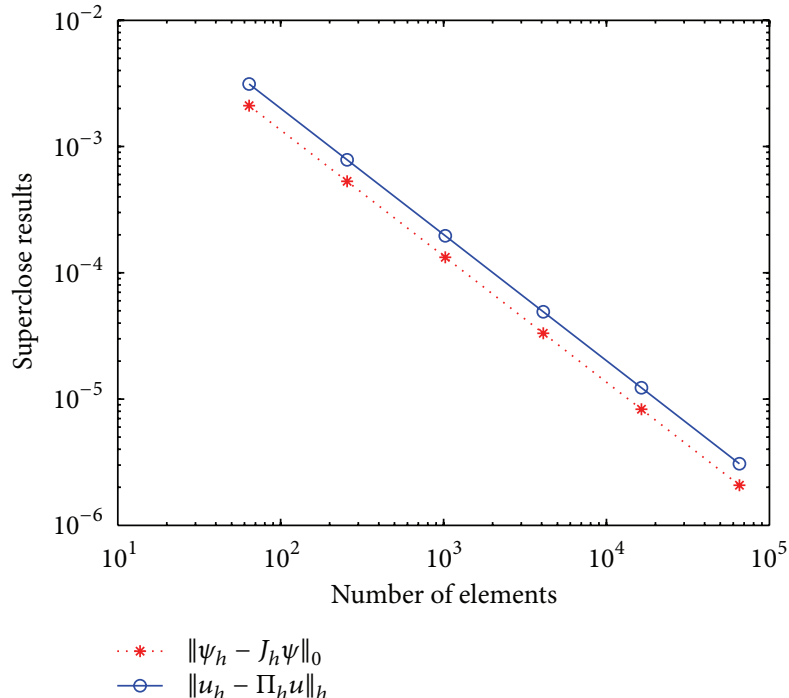

(a)

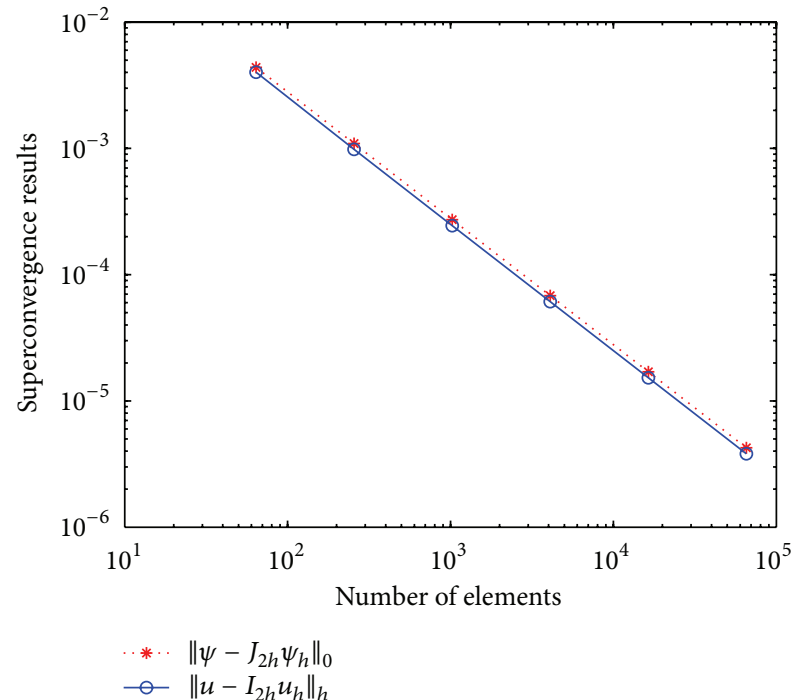

(b)

FiguRE 4: The superclose results (a) and the superconvergence results (b).

TABLE 1: Superclose results of $\psi$ and $u$.

\begin{tabular}{lcccc}
\hline$N$ & $\left\|\psi_{h}-J_{h} \psi\right\|_{0}$ & Rate & $\left\|u_{h}-\Pi_{h} u\right\|_{h}$ & Rate \\
\hline $8^{2}$ & 0.00210961611276 & $/$ & 0.00313196226739 & $/$ \\
$16^{2}$ & $5.305340499025525 e-004$ & 1.99146324331851 & $7.857858400185900 e-004$ & 1.99485875617198 \\
$32^{2}$ & $1.328284185506180 e-004$ & 1.99788150355682 & $1.966221092417072 e-004$ & 1.99871061529750 \\
$64^{2}$ & $3.321927509970684 e-005$ & 1.99947134660916 & $4.916651922457212 e-005$ & 1.99967742778650 \\
$128^{2}$ & $8.305579254650728 e-006$ & 1.99986789716594 & $1.229231701395274 e-005$ & 1.99991934319524 \\
$256^{2}$ & $2.076442340906698 e-006$ & 1.99996697808428 & $3.073122207277826 e-006$ & 1.99997983495344 \\
\hline
\end{tabular}

$I_{2 h}: u \in\left(H^{2}(\Omega) \cap H_{0}^{1}(\Omega)\right) \mapsto I_{2 h} u \in Q_{2}(\kappa)$ and $J_{2 h}:$ $\psi \in\left(H^{1}(\Omega)\right)^{2} \mapsto J_{2 h} \psi \in\left(Q_{1}(\kappa)\right)^{2}$ as $I_{2 h} u\left(a_{i}\right)=u\left(a_{i}\right)$, $i=1, \ldots, 9$, and $\int_{K_{j}}\left(\psi-J_{2 h} \psi\right)=0, j=1,2,3,4$, where $u\left(a_{i}\right)$ are the value of $u$ on the nodes $a_{i}$ and $a_{i}$ are nodes of $T_{h}$ on macroelement $\kappa$, while $\kappa \in T_{2 h}$ consists of the four small elements $K_{j}$ in $T_{h}(j=1,2,3,4)$, and $Q_{1}(\kappa)$ and $Q_{2}(\kappa)$ are bilinear and biquadratic piecewise polynomials spaces, respectively. It can be checked that the following properties hold:

$$
\begin{gathered}
I_{2 h} \Pi_{h} u=I_{2 h} u, \quad\left\|I_{2 h} u-u\right\|_{h} \leq C h^{2}|u|_{3}, \\
\left\|I_{2 h} v_{h}\right\|_{h} \leq C\left\|v_{h}\right\|_{h}, \quad \forall v_{h} \in M_{h}, \\
J_{2 h} J_{h} \psi=J_{2 h} \psi, \quad\left\|J_{2 h} \psi-\psi\right\|_{0} \leq C h^{2}|\psi|_{2}, \\
\left\|J_{2 h} \varphi_{h}\right\|_{0} \leq C\left\|\varphi_{h}\right\|_{0}, \quad \forall \varphi_{h} \in H_{h} .
\end{gathered}
$$

Then we can have the following superconvergence result.

Theorem 4. Under the assumptions in Theorem 3, there holds

$$
\left\|u-I_{2 h} u_{h}\right\|_{h}+\left\|\psi-J_{2 h} \psi_{h}\right\|_{0} \leq C h^{2}\left(\|u\|_{3}+\|\psi\|_{2}\right) .
$$

Proof. It follows from (30) in Theorem 3, (34), and the triangle inequality that

$$
\begin{aligned}
\left\|u-I_{2 h} u_{h}\right\|_{h}+\left\|\psi-J_{2 h} \psi_{h}\right\|_{0} \\
\leq\left\|u-I_{2 h} \Pi_{h} u\right\|_{h}+\left\|I_{2 h} \Pi_{h} u-I_{2 h} u_{h}\right\|_{h} \\
\quad+\left\|\psi-J_{2 h} J_{h} \psi\right\|_{0}+\left\|J_{2 h} J_{h} \psi-J_{2 h} \psi_{h}\right\|_{0} \\
\leq\left\|u-I_{2 h} \Pi_{h} u\right\|_{h}+\left\|I_{2 h}\left(\Pi_{h} u-u_{h}\right)\right\|_{h} \\
\quad+\left\|\psi-J_{2 h} J_{h} \psi\right\|_{0}+\|\| J_{2 h}\left(J_{h} \psi-\psi_{h}\right) \|_{0} \\
\leq\left\|u-I_{2 h} u\right\|_{h}+C\left\|\Pi_{h} u-u_{h}\right\|_{h} \\
\quad+\left\|\psi-J_{2 h} \psi\right\|_{0}+C\left\|J_{h} \psi-\psi_{h}\right\|_{0} \\
\leq C h^{2}\left(\|u\|_{3}+\|\psi\|_{2}\right),
\end{aligned}
$$

which is the desired result.

\section{Numerical Experiments}

In this section, some numerical examples and comparison with other methods are presented to confirm theoretical analysis and good performance of NMFEM. 
TABLE 2: Superconvergence results of $\psi$ and $u$.

\begin{tabular}{lcccc}
\hline$N$ & $\left\|\psi-J_{2 h} \psi_{h}\right\|_{0}$ & Rate & $\left\|u-I_{2 h} u_{h}\right\|_{h}$ & Rate \\
\hline $8^{2}$ & 0.00438686071544 & $/$ & 0.00401808068890 & $/$ \\
$16^{2}$ & 0.00109136640979 & 2.00705335488651 & $9.832140356840235 e-004$ & 2.03092911988780 \\
$32^{2}$ & $2.724757835149567 e-004$ & 2.00193562594041 & $2.445206258258704 e-004$ & 2.00754934612234 \\
$64^{2}$ & $6.809546234729317 e-005$ & 2.00049744480245 & $6.105100787093085 e-005$ & 2.00186914715453 \\
$128^{2}$ & $1.702238617992853 e-005$ & 2.00012537844145 & $1.525782546054283 e-005$ & 2.00046574790862 \\
$256^{2}$ & $4.255503868189354 e-006$ & 2.00003141881610 & $3.814148839722483 e-006$ & 2.00011631625361 \\
\hline
\end{tabular}

TABLE 3: Superclose results of $\psi$ and $u$.

\begin{tabular}{ccccc}
\hline$N$ & $\left\|\psi_{h}-J_{h} \psi\right\|_{0}$ & Rate & $\left\|u_{h}-\Pi_{h} u\right\|_{h}$ & Rate \\
\hline $8^{2}$ & 0.02493802431889 & $/$ & 0.03360261961598 & $/$ \\
$16^{2}$ & 0.00619633909611 & 2.00885917091448 & 0.00820721453088 & 2.03360913804615 \\
$32^{2}$ & 0.00154620480519 & 2.00268467156460 & 0.00203799536238 & 2.00974189629474 \\
$64^{2}$ & $3.863629284378830 e-004$ & 2.00070284735607 & $5.086068699851279 e-004$ & 2.00252791412788 \\
$128^{2}$ & $9.657883351384945 e-005$ & 2.00017773032582 & $1.270955080172888 e-004$ & 2.00063790775102 \\
$256^{2}$ & $2.414396265195381 e-005$ & 2.00004455935162 & $3.177035666820934 e-005$ & 2.00015984995534 \\
\hline
\end{tabular}

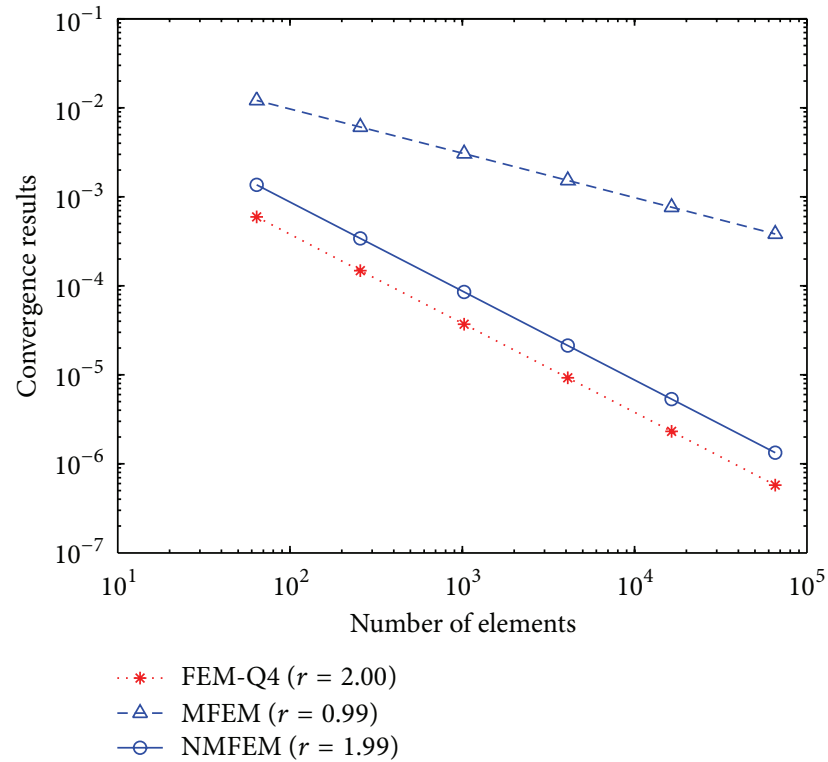

FIGURE 5: Convergence rates of $u$ in $L^{2}$ norm.

We consider the problem (1) with $\Omega=[0,1]^{2}$ and the exact solution is $u=x y(1-x)(1-y)$, and then the flux field $\psi=\left(\psi_{1}, \psi_{2}\right)$ can be expressed as $\psi_{1}=y(1-2 x)(1-y), \psi_{2}=$ $x(1-x)(1-2 y)$. We divide the domain $\Omega$ into a family of quasiuniform rectangles with number of $N$. The figures of exact solution $(u, \psi)$ of problem (1) and finite element approximation $\left(u_{h}, \psi_{h}\right)$ with $N=64^{2}$ are plotted in Figures 1, 2 , and 3 , respectively.

In Tables 1 and 2, we present the superclose and superconvergence results of the original variable $u$ in energy norm and flux $\psi$ in $L^{2}$ norm with $N=8^{2} ; 16^{2} ; 32^{2} ; 64^{2} ; 128^{2} ; 256^{2}$, respectively. It is clearly that $\left\|\psi_{h}-J_{h} \psi\right\|_{0},\left\|u_{h}-\Pi_{h} u\right\|_{h}$, $\left\|\psi-J_{2 h} \psi_{h}\right\|_{0}$, and $\left\|u-I_{2 h} u_{h}\right\|_{h}$ are converged at order 2 with

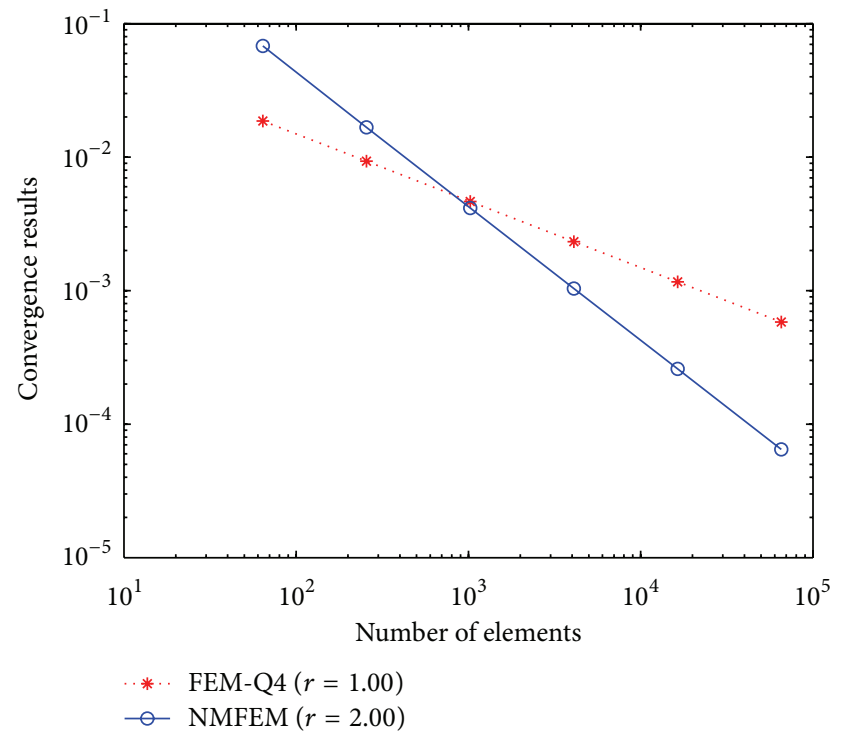

FIGURE 6: Convergence rates of $u$ in energy norm.

respect to $h$, which coincide with our theoretical analysis in Theorems 3 and 4 . In order to describe the results more intuitively, we plot the errors in the logarithm scales in Figure 4.

Moreover, we compare the results of NMFEM with those of FEM using 4-node quadrilateral (FEM-Q4) and MFEM ( $u$ and flux $\psi$ are approximated by piecewise constants and the Raviart-Thomas element, resp.).

The convergence rates of errors of $u$ in $L^{2}$ and energy norm are shown in Figures 5 and 6. The comparison of the flux $\psi$ in $L^{2}$ norm of NMFEM with MFEM is also given in Figure 7 . It is observed that (a) the convergence rates of $u$ and flux $\psi$ in $L^{2}$ norm of NMFEM are better than those of MFEM; 
TABLE 4: Superconvergence results of $\psi$ and $u$.

\begin{tabular}{lccrc}
\hline$N$ & $\left\|\psi-J_{2 h} \psi_{h}\right\|_{0}$ & Rate & $\left\|u-I_{2 h} u_{h}\right\|_{h}$ & Rate \\
\hline $8^{2}$ & 0.07704230005041 & $/$ & 0.06805851358971 & $/$ \\
$16^{2}$ & 0.01905713318621 & 2.01531966736879 & 0.01670893020781 & 2.02615627537278 \\
$32^{2}$ & 0.00474917374465 & 2.00458266513090 & 0.00415565843089 & 2.00747038382646 \\
$64^{2}$ & 0.00118603209709 & 2.00153348351974 & 0.00103721683378 & 2.00235955252584 \\
$128^{2}$ & $2.964169140807930 e-004$ & 2.00044337559203 & $2.5918444848686839 e-004$ & 2.00066626183030 \\
$256^{2}$ & $7.409813186219603 e-005$ & 2.00011869741141 & $6.478819154402888 e-005$ & 2.00017656612731 \\
\hline
\end{tabular}

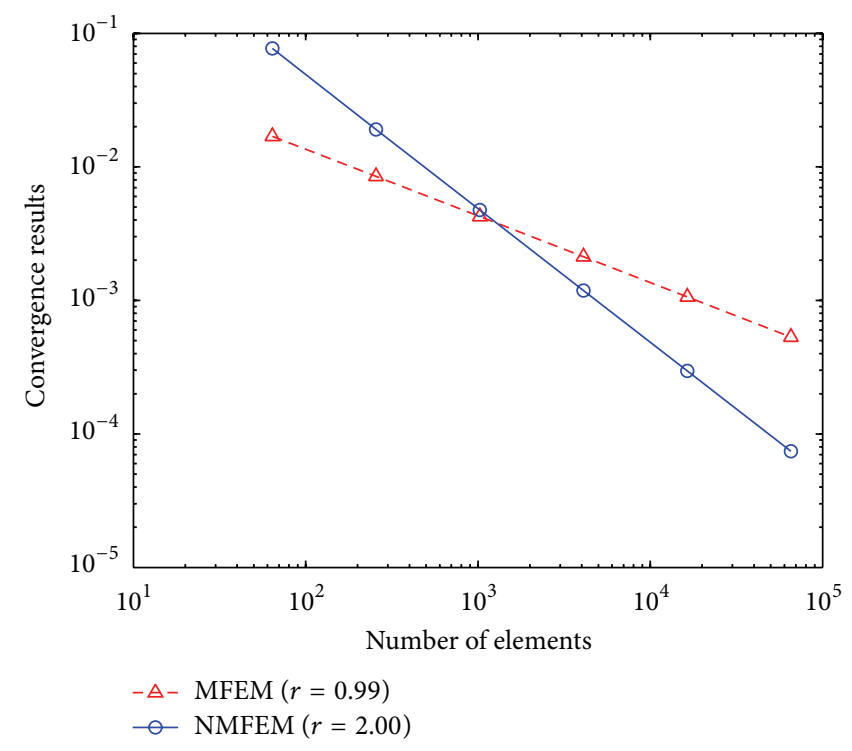

FIgURE 7: Convergence rates of the flux $\psi$ in $L^{2}$ norm.

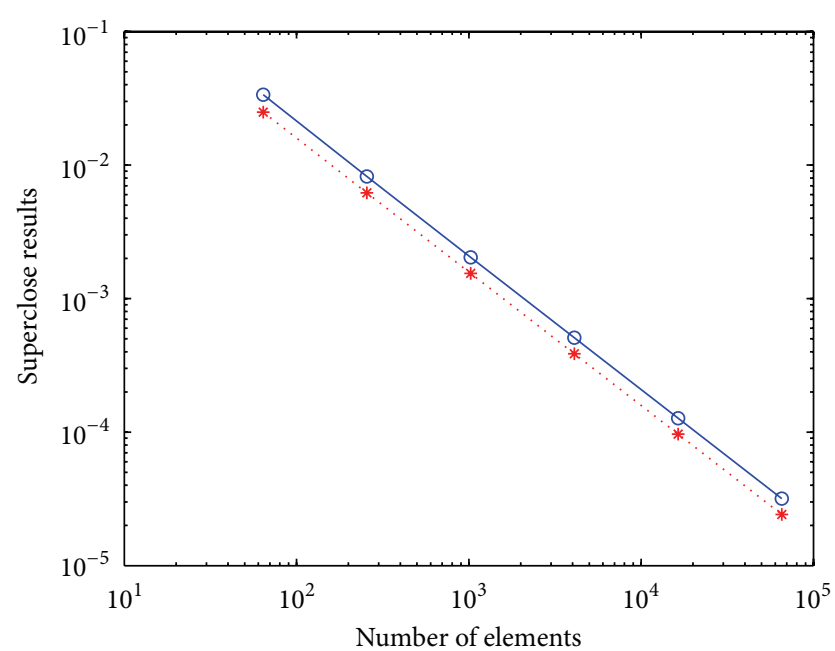

..*.. $\left\|\psi_{h}-J_{h} \psi\right\|_{0}$

$\multimap\left\|u_{h}-\Pi_{h} u\right\|_{h}$

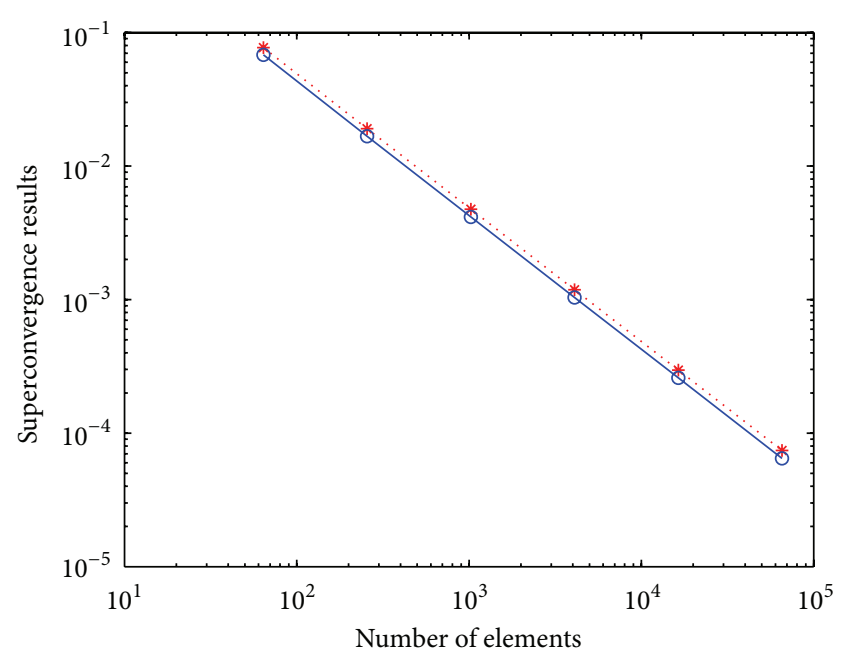

..*.. $\left\|\psi-J_{2 h} \psi_{h}\right\|_{0}$

$\bigcirc\left\|u-I_{2 h} u_{h}\right\|_{h}$

(a)

(b)

FIGURE 8: The superclose results (a) and the superconvergence results (b). 
(b) NMFEM has almost the same rate as FEM-Q4 for $u$ in $L^{2}$ norm, but a higher rate than FEM-Q4 for $u$ in energy norm.

Furthermore, a numerical experiment is carried out to demonstrate the effectiveness and accuracy of NMFEM for the following diffusion problem:

$$
\begin{gathered}
-\operatorname{div}(D \nabla u)=f, \quad \text { in } \Omega, \\
u=u_{\partial}, \quad \text { on } \partial \Omega,
\end{gathered}
$$

where $f$ is the source term and $f \in L^{2}(\Omega), u_{\partial}$ is the boundary data, the permeability $D$ is a symmetric tensorvalued function such that (a) $D$ is piecewise Lipschitzcontinuous on $\Omega$ and (b) the set of the eigenvalues of $D$ is included in $\left[\lambda_{\text {min }}, \lambda_{\text {max }}\right]$ (with $\lambda_{\text {min }}>0$ ) for all $(x, y) \in \Omega$.

As [13], we consider the problem (37) with $\Omega=[0,1]^{2}$,

$$
\begin{gathered}
D=\left(\begin{array}{ll}
1 & 0 \\
0 & 1
\end{array}\right) \quad \text { if } x \leq 0.5, \\
D=\left(\begin{array}{cc}
100 & 0 \\
0 & 0.01
\end{array}\right) \quad \text { if } x>0.5,
\end{gathered}
$$

and the analytical solution

$$
\begin{gathered}
u=\cos (\pi x) \sin (\pi y) \quad \text { if } x \leq 0.5, \\
u=0.01 \cos (\pi x) \sin (\pi y) \quad \text { if } x>0.5 .
\end{gathered}
$$

Let $\psi=D \nabla u$, we can get the superclose and superconvergence results of $u$ in energy norm and flux $\psi$ in $L^{2}$ norm. The errors are listed in Tables 3 and 4 and plotted in the logarithm scales in Figure 8, respectively.

\section{Acknowledgments}

The authors would like to express their sincere thanks to the anonymous referee for his many helpful suggestions, which contribute significantly to the improvement of the paper. The research is supported by the NSF of China (no. 10971203; no. 11271340), Research Fund for the Doctoral Program of Higher Education of China (no. 20094101110006), and Foundation of He'nan Educational Committee (no. 13B110144).

\section{References}

[1] P.-A. Raviart and J. M. Thomas, "A mixed finite element method for 2nd order elliptic problems," in Mathematical Aspects of Finite Element Methods, vol. 606 of Lecture Notes in Mathematics, pp. 292-315, Springer, Berlin, Germany, 1977.

[2] M. Farhloul and M. Fortin, "A nonconforming mixed finite element for second-order elliptic problems," Numerical Methods for Partial Differential Equations, vol. 13, no. 5, pp. 445-457, 1997.

[3] B. Cockburn, G. Kanschat, I. Perugia, and D. Schötzau, "Superconvergence of the local discontinuous Galerkin method for elliptic problems on Cartesian grids," SIAM Journal on Numerical Analysis, vol. 39, no. 1, pp. 264-285, 2001.

[4] Z.-D. Luo, "Method of nonconforming mixed finite element for second order elliptic problems," Journal of Computational Mathematics, vol. 18, no. 5, pp. 449-456, 2000.
[5] D. Y. Shi and C. X. Wang, "A new low-order non-conforming mixed finite-element scheme for second-order elliptic problems," International Journal of Computer Mathematics, vol. 88, no. 10, pp. 2167-2177, 2011.

[6] F. Brezzi and M. Fortin, Mixed and Hybrid Finite Element Methods, vol. 15 of Springer Series in Computational Mathematics, Springer, New York, NY, USA, 1991.

[7] P. B. Bochev and M. D. Gunzburger, "Finite element methods of least-squares type," SIAM Review, vol. 40, no. 4, pp. 789-837, 1998.

[8] P. B. Bochev, C. R. Dohrmann, and M. D. Gunzburger, "Stabilization of low-order mixed finite elements for the Stokes equations," SIAM Journal on Numerical Analysis, vol. 44, no. 1, pp. 82-101, 2006.

[9] D.-Y. Shi and H.-H. Wang, "Nonconforming $H^{1}$-Galerkin mixed FEM for Sobolev equations on anisotropic meshes," Acta Mathematicae Applicatae Sinica, vol. 25, no. 2, pp. 335-344, 2009.

[10] S. C. Chen and H. R. Chen, "New mixed element schemes for a second-order elliptic problem," Mathematica Numerica Sinica, vol. 32, no. 2, pp. 213-218, 2010.

[11] F. Shi, J. P. Yu, and K. T. Li, "A new mixed finite element scheme for elliptic equations," Chinese Journal of Engineering Mathematics, vol. 28, no. 2, pp. 231-237, 2011.

[12] J. Hu, H. Y. Man, and Z. C. Shi, "Constrained nonconforming rotated $Q_{1}$ element for Stokes flow and planar elasticity," Mathematica Numerica Sinica, vol. 27, no. 3, pp. 311-324, 2005.

[13] J. Droniou and C. le Potier, "Construction and convergence study of schemes preserving the elliptic local maximum principle," SIAM Journal on Numerical Analysis, vol. 49, no. 2, pp. 459-490, 2011.

[14] R. Rannacher and S. Turek, "Simple nonconforming quadrilateral Stokes element," Numerical Methods for Partial Differential Equations, vol. 8, no. 2, pp. 97-111, 1992.

[15] D. Y. Shi, Y. C. Peng, and S. C. Chen, "Error estimates for rotated $Q_{1}^{\text {rot }}$ element approximation of the eigenvalue problem on anisotropic meshes," Applied Mathematics Letters, vol. 22, no. 6, pp. 952-959, 2009.

[16] Q. Lin and N. N. Yan, The Construction and Analysis of High Accurate Finite Element Methods, Hebei University Press, Baoding, China, 1996. 


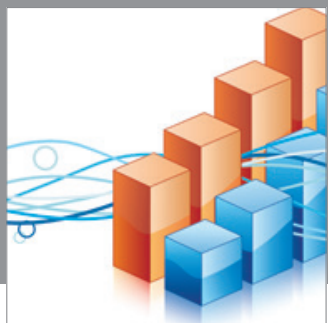

Advances in

Operations Research

mansans

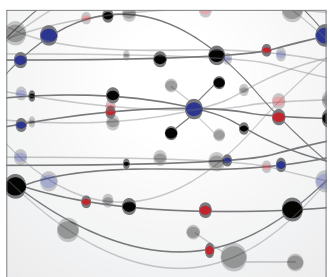

The Scientific World Journal
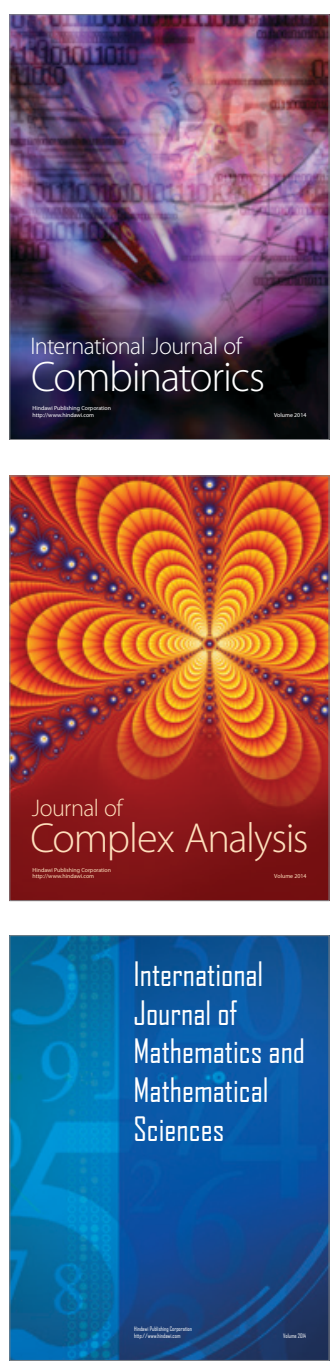
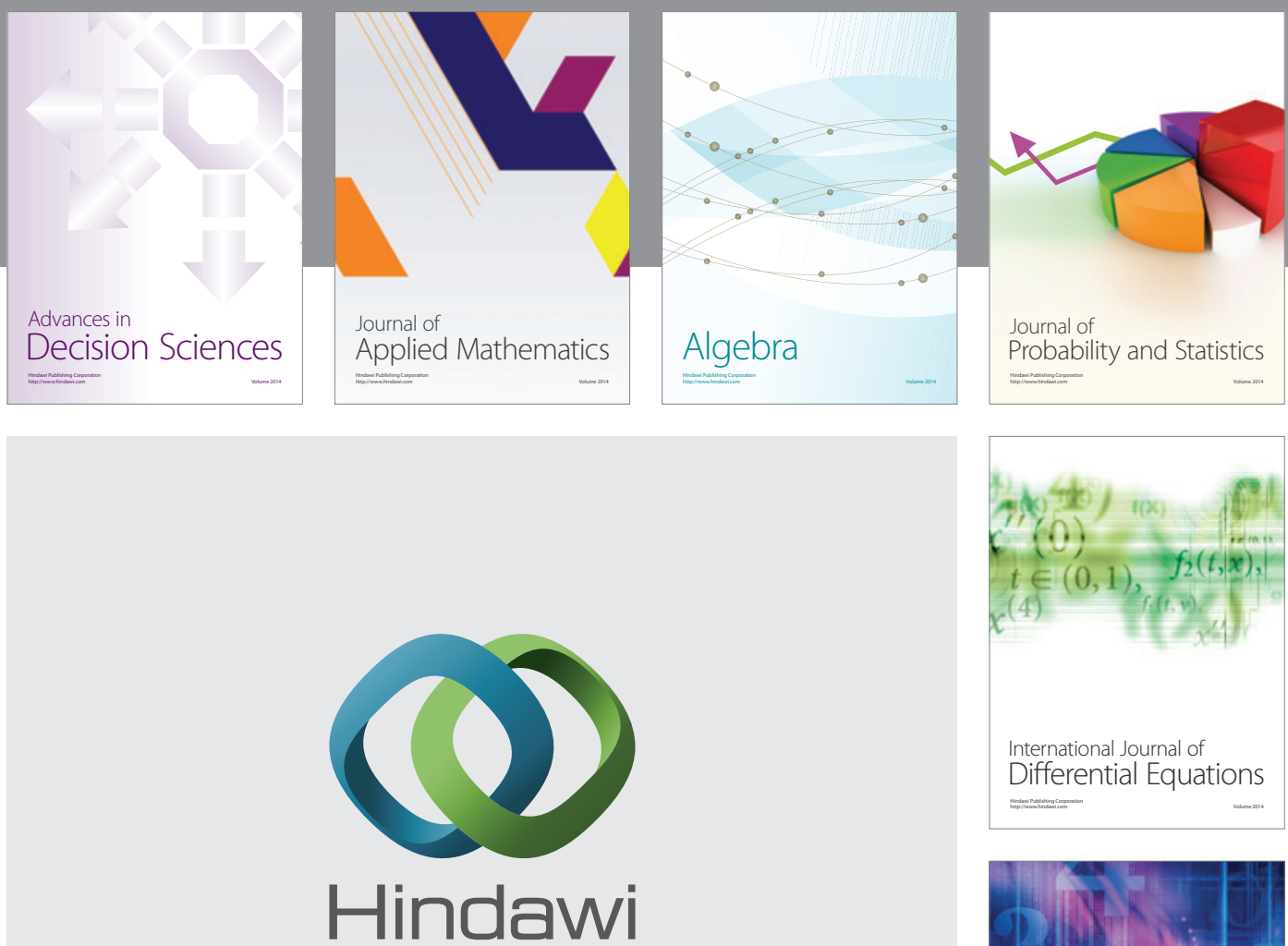

Submit your manuscripts at http://www.hindawi.com
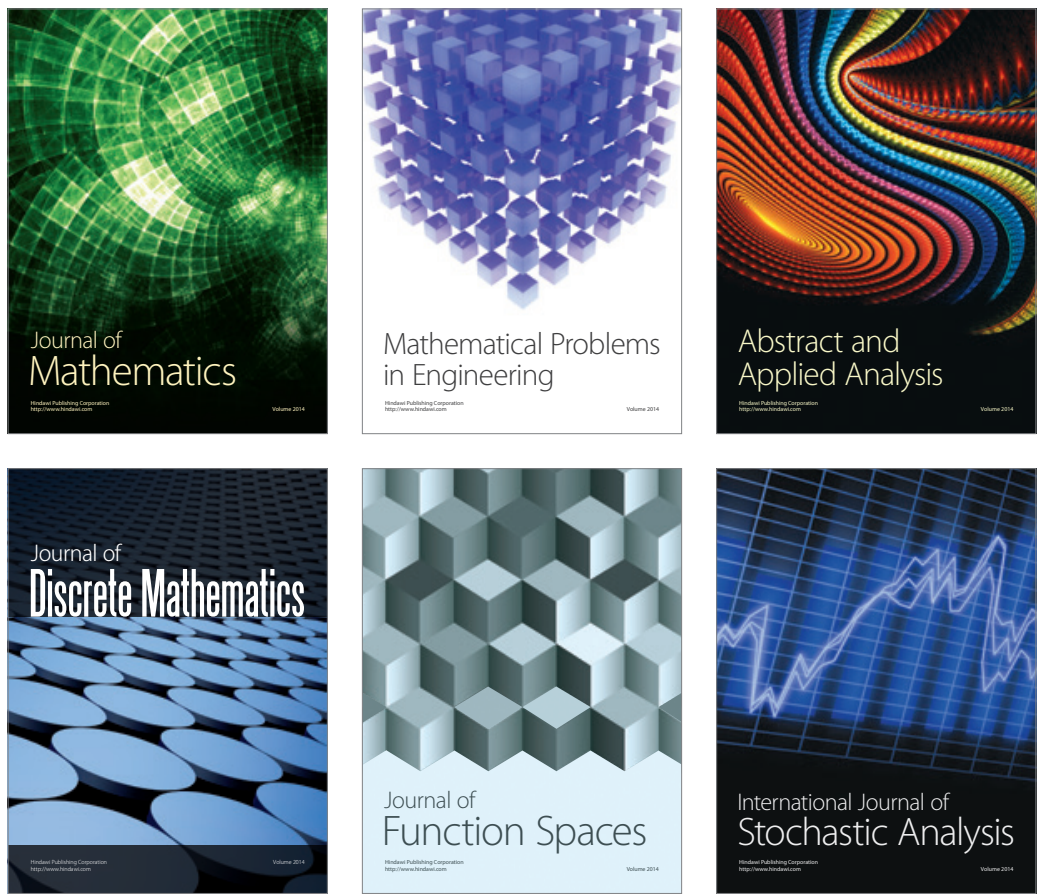

Journal of

Function Spaces

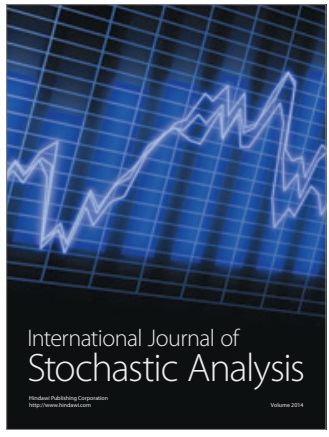

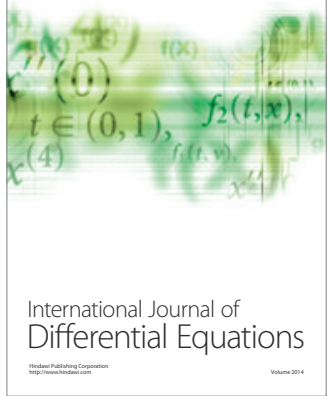
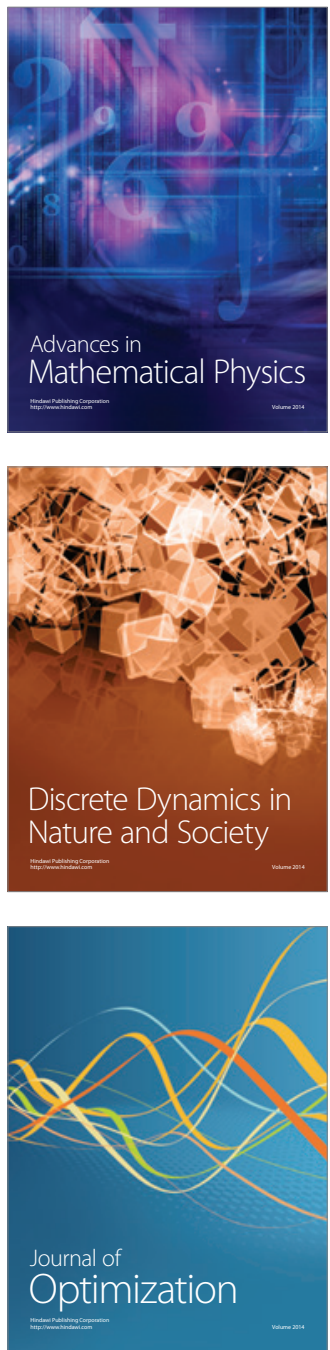